\title{
Detection of change in nonstationary binary sequences*
}

\author{
JOHN THEIOS, University of Wisconsin, Madison, Wisconsin 53706 \\ JOHN W. BRELSFORD, JR., Rice University, Houston, Texas 77001 \\ and
}

PHYLLIS RYAN, University of Pennsylvania, Philadelphia, Pennsylvania 19104

The present study was designed to investigate the ability of humans to detect changes in the underlying probability structure of binary sequences of events. Four Ss were presented with sequences of 30 events (zeros and ones) which had been generated by two underlying probability values. $P_{1}$ was the probability of a one in the first $i$ events, $P_{2}$ was the probability of a one in the last 30-i events, and the distribution of $i$ was uniform from 1 to 29 . The Ss indicated, without feedback, where the transition point from $P_{1}$ to $P_{2}$ took place for four sets of problems in which $P_{1}$ and $P_{2}$ took on the following pairs of values: $(.40, .60),(.20, .80),(.05, .60)$, and $(.50,1.00)$. Estimates by Ss were compared with true transition points, $i$, and with maximum likelihood estimates of $i$. The difference between $P_{1}$ and $P_{2}$ and the closeness of at least one $P$ value to the boundaries, either zero or unity, interacted in determining the difficulty of the problem for Ss.

In many situations human Ss are required to monitor the output of a stationary process, but must perform some act whenever they detect that the relative frequencies of the output events have changed. For purposes of experimental investigation, the requirements of this type of detection task may be realized in various ways. For example, an on-line computer may be programmed to first select two probability values, $P_{1}$ and $P_{2}$, and an integer, $\mathrm{i}$, and then generate and display a sequence of $\mathrm{N}$ binary events (e.g., zeros and ones). $P_{1}$ is the probability of each of the first $i$ events being a one, whereas $P_{2}$ is the probability of the remaining $\mathrm{N}-\mathrm{i}$ events being one. The S's task is to estimate the point in the sequence at which the underlying probabilities changed from $P_{1}$ to $P_{2}$. In situations in which the information is presented to the $\mathrm{S}$ serially, the optimal decision strategy is to revise sequentially the estimate of the current probability, using some form of Bayes's theorem. Robinson (1964) and Chinnis and Peterson (1968) have investigated behavior in this serial task.

However, when all the information is displayed to the $\mathrm{S}$ simultaneously, an optimal decision strategy is to respond

\footnotetext{
*This research was conducted while the first author was a National Science Foundation Science Faculty Fellow at the Institute for Mathematical Studies in the Social Sciences at Stanford University. We wish to express our appreciation to Professors Patrick Suppes and Richard C. Atkinson, who so generously made their Computer-Based Learning Laboratory available to us. This research was supported in part by PHS Research Grants HD 02212-03, HD 003540-01, MH 19158-01, and MH 1671-01 from the National Institutes of Heilth, USPHS. Reprint requests should be sent to John Theios, Department of Psychology, University of Wisconsin, Madison, Wisconsin 53706.
}

Perception? I' vchophysics, 1971, Vol. 9 (6)

with an estimate of the transition point which maximizes the likelihood of the entire sequence. Theios (1968) has presented a method for determining maximum likelihood estimates of the parameters of a process generating such a sequence of binary events. The likelihood of any particular binary sequence of $\mathrm{N}$ events is:

$L=\left(P_{1}\right)^{x}\left(1-P_{1}\right)^{i-x}\left(P_{2}\right)^{y}\left(1-P_{2}\right)^{N-i-y}$

In Eq. 1, $x$ represents the number of ones in the first $i$ events and $y$ represents the number of ones in the last $\mathbf{N}-\mathrm{i}$ events. Given any $i$, the maximum likelihood estimates of $P_{1}$ and $P_{2}$ are just the proportion of ones in the first $i$ trials and the last $(\mathrm{N}-\mathrm{i})$ trials, respectively:

$$
\begin{aligned}
& P_{1}=x / i \\
& P_{2}=x /(N-i) .
\end{aligned}
$$

To obtain the maximum of the likelihood function, one merely uses a computer to select the value of $i$ that makes the value of Eq. 1 as large as possible.

We thus have a normative model which, given the statistical information in the event sequence, dictates the optimal decision to be made. The present investigation was an attempt to determine how close humans come to making maximum likelihood estimates in situations of this type over a variety of event probabilities.

Intuitively, it would seem that the difficulty of estimating a transition point in a binary sequence would be a function of the difference between the two probability values. Detection should be easier the greater the difference between $P_{1}$ and $P_{2}$, and vice versa. Further, to the degree that one or both of the probabilities are near zero or one, the estimation task should become easier since the variance in the events will be minimal in such cases. With these intuitions in mind, four problems were selected for investigation. Two of the problems were symmetric about the center of the unit interval. In the first problem the initial probability $\left(\mathrm{P}_{1}\right)$ was .40 and the terminal probability $\left(\mathrm{P}_{2}\right)$ was .60 . In the second problem, $P_{1}$ was .20 and $\mathrm{P}_{2}$ was 80 . In the asymmetric conditions, the third problem had $P_{1}=.05$ and $P_{2}=.60$, while the fourth problem had $P_{1}=.50$ and $P_{2}=1.00$.

\section{METHOD}

The Ss were four Stanford University undergraduates who were paid $\$ 2$ for each of at least 16 daily experimental sessions. Event sequences were generated by a time-sharing program running in a Digital Equipment Corporation PDP-1 computer and displayed on Philco Read cathode ray tubes (CRT).

Each $S$ was run concurrently on four problems in which the probability pairs were $(.40, .60),(.20, .80),(.05, .60)$, and $(.50,1.00)$. On each decision trial, the computer was programmed to first select one of the four problems at random, with the constraint that problems with identical underlying probability pairs did not occur on adjacent trials. The computer selected at random an integer, $i$, from a uniform distribution from 1 to 29 . The selected integer was set to be the transition trial, following which the event-generating probability changed from $P_{1}$ to $P_{2}$. The computer then generated a sequence of 30 zeros and ones, according to the selected $i$, $P_{1}$, and $P_{2}$ values. The sequence was displayed in its entirety on the CRT. Each $S$ was instructed to touch, with his light pen, the last event he felt was generated with probability $P_{1}$. Before the experiment the Ss had been given a short lecture on probability theory, including a discussion of the manner in which the sequences would be generated by the computer. Effectively, Ss were told that their task was to divide the sequence into two parts at a point where they thought it naturally should be divided. As soon as the sequence had been generated, the computer calculated the maximum likelihood estimate of the trial of transition and computed the difference between the maximum likelihood estimate and the true transition integer. After $S$ responded, the machine computed the differences between S's estimate and the maximum likelihood estimate.

No feedback as to the true transition point or the maximum likelihood estimate 


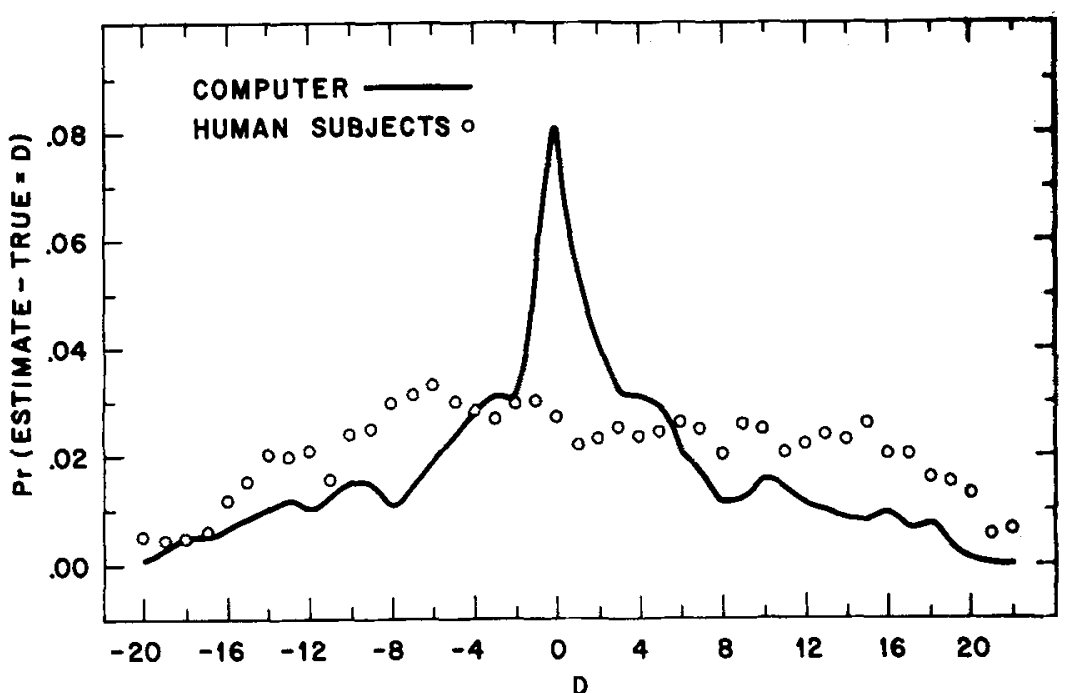

Fig. 1. Probability distributions of the differences between the true trial of transition from $P_{1}$ to $P_{2}$ and (a) the $S$ 's estimate of that trial and (b) computer-generated maximum likelihood estimates of that trial: $P_{1}=.40$ and $P_{2}=.60$.

of the transition point was given to the Ss. Ten seconds following S's response, the sequence for the next problem was displayed.

Each S was run on 120 problems per day for at least 16 daily sessions. Each session was scheduled for $50 \mathrm{~min}$, and $\mathrm{S}$ worked at his own pace, usually finishing in somewhat less than $50 \mathrm{~min}$. The first few days of testing were considered as orientation to the task; hence, the data from several initial sessions have not been considered in this report. Further, in that the reliability of a maximum likelihood estimate is an increasing function of the sample size, the computer was programmed to ignore the data on problems where the true transition point was less than 6 or greater than 24. In most cases this restriction ensured that the maximum likelihood estimate was based upon more than just one or two observations.

Figure 1 presents the obtained distributions of differences for the first problem in which $P_{1}$ was .40 and $P_{2}$ was 60. Each distribution is based upon 7,248 observations. The solid line represents an obtained sampling distribution of differences between the maximum likelihood estimate and the true point of transition. It can be seen that the humans are performing much more poorly than maximum likelihood performance. The individual chi squares of goodness of fit between the human and maximum likelihood distribution of differences are given in Table 1. The sum of the four chi squares is 3,127 , with $160 \mathrm{df}$. The Pearson $r$ among the S's judgments, the maximum likelihood estimates, and the true trials of transition are given in Table 1.
Table 1 , and the total chi square is 666 , with $112 \mathrm{df}$. The correlations in Table 1 for the $.20-80$ problem indicate that, on the average, the maximum likelihood estimates correlate .68 with the true values, while the human estimates correlate about .45 with the true values. The average correlation between the human and the machine (maximum likelihood) estimates is .48 .

The results for the $.05-.60$ problem $(\mathrm{N}=7,240)$ are presented in Fig. 3. Even though the difference between .60 and .05 is less than the difference between .80 and .20 , both the machine and the humans are doing better than on the $.20-.80$ problem $\left[\chi^{2}(112)=597\right]$. This is probably because .05 is so close to zero that the initial events of the sequence will consist almost entirely of zeros. Thus, a shift to one or more ones is highly detectable. The correlations in Table 1 for the $.05-.60$ problem are generally higher than those of the previous problems. The average correlation between the maximum likelihood estimate and the true value is 86 , and the average correlation between the human estimates and the true value is about .71 . With this easier problem, the human estimates are beginning to approximate the maximum likelihood estimates, in that they correlate about .74. It should specifically be noted that the sampling distribution of the differences between the maximum likelihood estimates and the true transition points is neither normal nor symmetric.

The results for the $.50-1.00$ problem $(\mathrm{N}=7,249)$ are presented in Fig. 4. It can be seen that in this problem both the human and the maximum likelihood estimates are quite close to the true

Table 1

Pearson Product Moment Correlation Coefficients (I) and Goodness-of-Fit Chi Squares $\left(\chi^{2}\right)$

\begin{tabular}{|c|c|c|c|c|c|c|c|c|}
\hline \multirow[b]{2}{*}{ Problem } & \multirow[b]{2}{*}{$\mathbf{P}_{1}$} & \multirow[b]{2}{*}{$\mathbf{P}_{2}$} & \multirow[b]{2}{*}{$\mathrm{S}$} & \multirow{2}{*}{$\begin{array}{c}\text { Human- } \\
\text { True } \\
\mathrm{I} \\
\end{array}$} & \multirow{2}{*}{$\begin{array}{c}\text { Machine- } \\
\text { True } \\
r\end{array}$} & \multicolumn{3}{|c|}{ Human-Machine } \\
\hline & & & & & & $r$ & $x^{2}$ & $\mathrm{~d} \mathbf{f}$ \\
\hline \multirow[t]{2}{*}{$\mathbf{I}$} & .40 & .60 & $\begin{array}{l}1 \\
2 \\
3 \\
4\end{array}$ & $\begin{array}{l}.21 \\
.30 \\
.33 \\
.25\end{array}$ & $\begin{array}{l}.38 \\
.39 \\
.42 \\
.37\end{array}$ & $\begin{array}{l}.41 \\
.33 \\
.30 \\
.34\end{array}$ & $\begin{array}{l}985 \\
628 \\
756 \\
758\end{array}$ & $\begin{array}{l}40 \\
40 \\
40 \\
40\end{array}$ \\
\hline & & & Average & .27 & .39 & .34 & 782 & 40 \\
\hline \multirow[t]{2}{*}{ II } & .20 & .80 & $\begin{array}{l}1 \\
2 \\
3 \\
4\end{array}$ & $\begin{array}{l}.49 \\
.50 \\
.46 \\
.37\end{array}$ & $\begin{array}{l}.61 \\
.64 \\
.75 \\
.72\end{array}$ & $\begin{array}{l}.51 \\
.55 \\
.42 \\
.43\end{array}$ & $\begin{array}{r}193 \\
175 \\
94 \\
204\end{array}$ & $\begin{array}{l}28 \\
28 \\
28 \\
28\end{array}$ \\
\hline & & & Average & .45 & .68 & .48 & 167 & 28 \\
\hline \multirow[t]{2}{*}{ III } & .05 & .60 & $\begin{array}{l}1 \\
2 \\
3 \\
4\end{array}$ & $\begin{array}{l}.73 \\
.70 \\
.72 \\
.70\end{array}$ & $\begin{array}{l}.83 \\
.82 \\
.91 \\
.88\end{array}$ & $\begin{array}{l}.72 \\
.70 \\
.81 \\
.75\end{array}$ & $\begin{array}{r}246 \\
106 \\
92 \\
153\end{array}$ & $\begin{array}{l}34 \\
34 \\
34 \\
34\end{array}$ \\
\hline & & & Average & .71 & .86 & .74 & 149 & 34 \\
\hline \multirow[t]{2}{*}{ IV } & .50 & 1.00 & $\begin{array}{l}1 \\
2 \\
3 \\
4\end{array}$ & $\begin{array}{l}.90 \\
.91 \\
.93 \\
.92\end{array}$ & $\begin{array}{l}.96 \\
.98 \\
.94 \\
.96\end{array}$ & $\begin{array}{l}.90 \\
.81 \\
.93 \\
.93\end{array}$ & $\begin{array}{l}64 \\
29 \\
12 \\
32\end{array}$ & $\begin{array}{l}5 \\
5 \\
5 \\
5\end{array}$ \\
\hline & & & Average & .92 & .96 & .89 & 34 & 5 \\
\hline
\end{tabular}




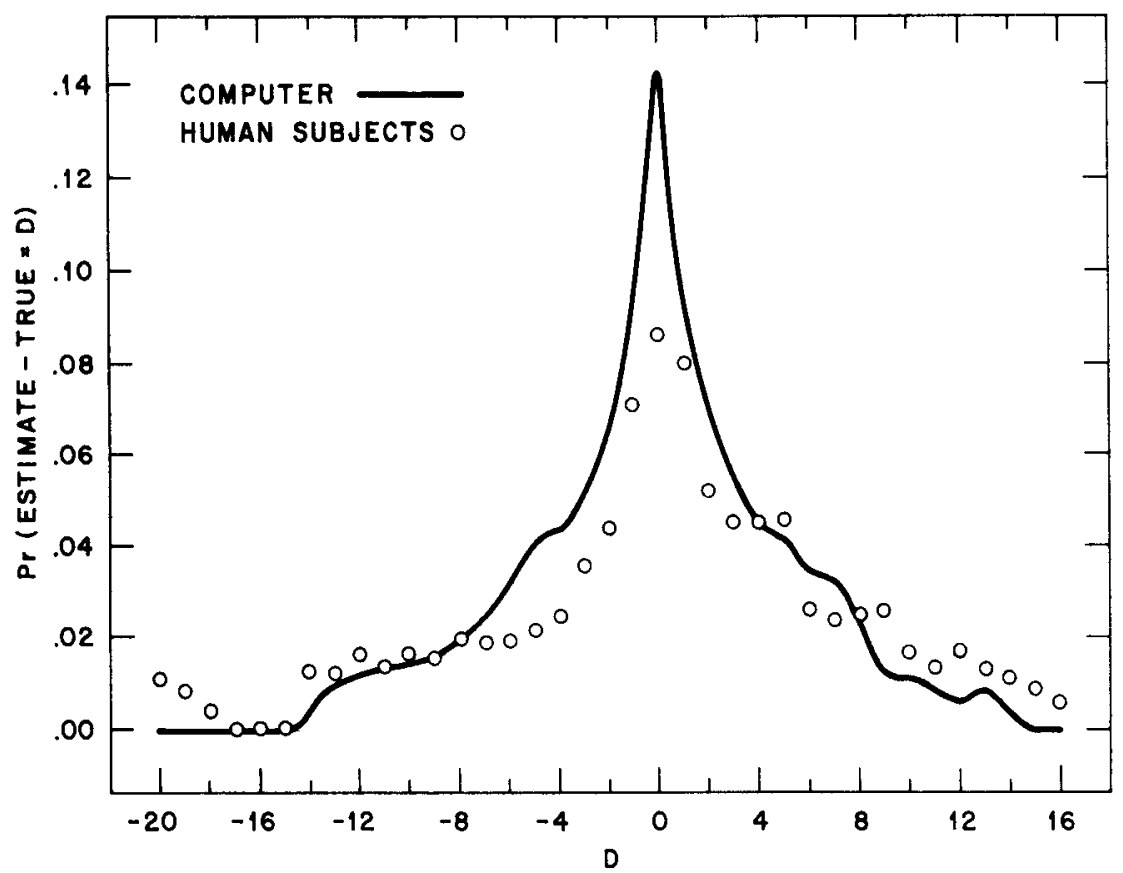

Fig. 2. Probability distributions of the differences between the true trial of transition from $P_{1}$ to $P_{2}$ and (a) the $S$ 's estimate of that trial and (b) computer-generated maximum likelihood estimates of that trial: $P_{1}=.20$ and $P_{2}=.80$.

transition point and that the human estimates are approximating the maximum likelihood estimates to a reasonable degree $\left[\chi^{2}(20)=138\right]$. Most of the correlations given in Table 1 for the $.50-1.00$ problem are in the $.90 \mathrm{~s}$, indicating close agreement among maximum likelihood estimates, human estimates, and true values.

Finally, it can be strikingly seen in Fig. 4

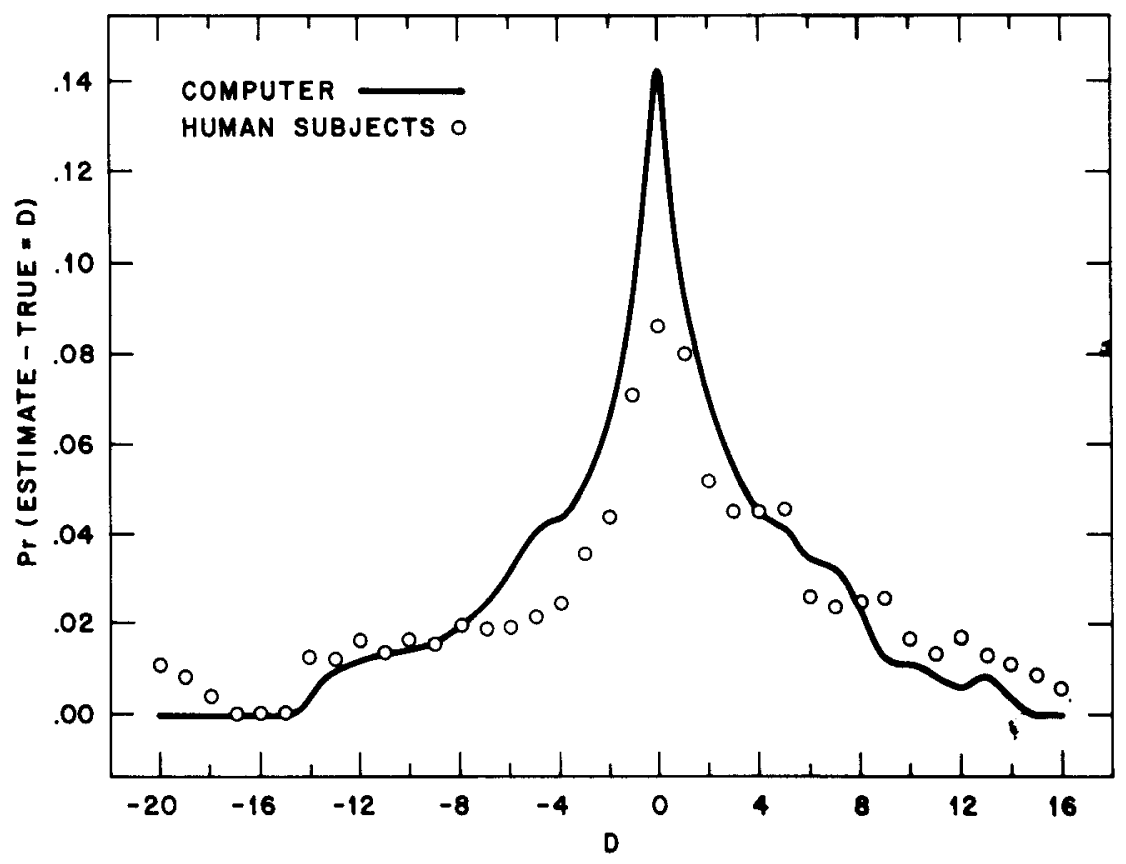

Fig. 3 Probability distributions of the differences between the true trial of transition from $P_{1}$ to $P_{2}$ and (a) the $S$ 's estimate of that trial and (b) computer-generated maximum likelihood estimates of that trial: $P_{1}=.05$ and $P_{2}=.60$. differences between the maximum likelihood estimate and the true transition point for the $.50-1.00$ problem is completely asymmetric, having all of its density on the negative side of the error scale. The means and standard deviations of various differences present a concise summary of the general trends in the data

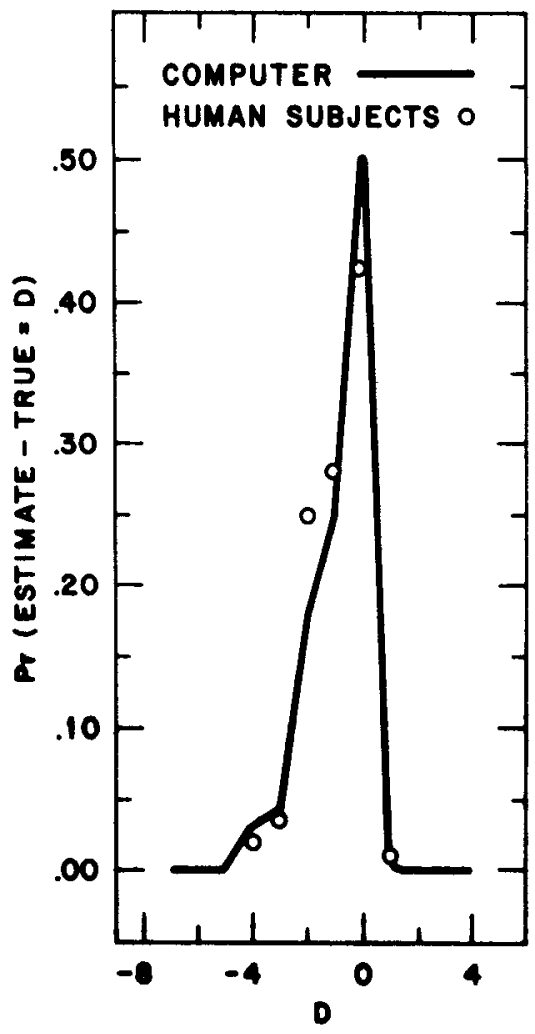

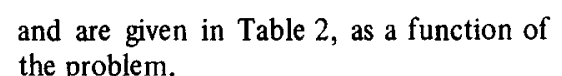
the problem.

\section{DISCUSSION}

On the basis of the results, it is obvious that adult human estimates of the point of change in the underlying probabilities of nonstationary binary sequences approximate maximum likelihood estimates only for the most trivial problems. Two variables seem to interact in determining the difficulty of the problem for the $S$. These are (1) the difference between $P_{1}$ and $P_{2}$, and (2) the closeness of at least one of the probabilities to the boundaries, either zero or unity. These two variables both enter into a determination of the expected amount of variability (or uncertainty) in the sequences of events. It appears that the difficulty of the detection problem for the $S$ is directly related to the amount of variability (and, thus, the amount of uncertainty) in the event sequences.

It should be remembered that in the present experiment the $S s$ received no information feedback about their performance. The question naturally arises as to how much the performance of the $S$ s 
Table 2

Means (Bias*) and Standard Deviations (Error) of Differences

\begin{tabular}{|c|c|c|c|c|c|c|c|}
\hline \multirow[b]{2}{*}{ Problem } & \multirow[b]{2}{*}{$\mathbf{P}_{1}$} & \multirow[b]{2}{*}{$P_{2}$} & \multicolumn{2}{|c|}{ Human-True } & \multicolumn{2}{|c|}{ Machine-True } & \multirow{2}{*}{$\begin{array}{c}\begin{array}{c}\text { Observa- } \\
\text { vations }\end{array} \\
\mathrm{N}\end{array}$} \\
\hline & & & $\mathbf{M}$ & SD & $\mathrm{M}$ & SD & \\
\hline I & .40 & .60 & 3.28 & 9.26 & .93 & 5.21 & 7,248 \\
\hline II & .20 & .80 & 2.07 & 5.21 & .63 & 4.23 & 7,245 \\
\hline III & .05 & .60 & -.92 & 3.21 & 1.21 & 3.07 & 7,240 \\
\hline IV & .50 & 1.00 & -.99 & .82 & -1.22 & .87 & 7,249 \\
\hline
\end{tabular}

*A negative mean indicates the point of change is underestimated. $A$ positive mean indicates the point of change is overestimated.

could be improved by providing them with feedback about the appropriateness of their responses. It appears that the effect of three different feedback conditions could be investigated: (1) feedback about the true point of transition, (2) feedback about the maximum likelihood estimate of the point of transition, and (3) feedback about both the true transition point and the maximum likelihood estimate. Our intuition is that feedback about the true transition alone would lead to poorer performance than would either of the two conditions that provide information about given true information feedback would ever converge to a maximum likelihood decision strategy. A further interesting question is whether feedback about the true transition point, coupled with feedback about the maximum likelihood estimate, would result in improvement of the S's behavior over that of maximum likelihood feedback by itself. It is possible that the addition of the true feedback could act as "noise" and thus attenuate the effectiveness of the maximum likelihood feedback.

\section{REFERENCES}

CHINNIS, J. O, JR., \& PETERSON, C. R. Inference about a nonstationary process. Journal of Experimental Psychology, 1968, $77,620-625$

ROBINSON, G. H. Continuous estimation of a time-varying probability. Ergonomics, 1964, 7 , $7-21$.

THEIOS, J. Finite integer models for learning in individual subjects. Psychological Review, $1968,75,292-307$

(Accepted for publication October 30, 1970.) 